ARTÍCULO DE INVESTIGACIÓN

\title{
Características Clínicas y Demográficas de la Enfermedad Celíaca en Panamá
}

[Clinical and Demographic Characteristics of Celiac Disease in Panama]

Dr. Ricardo Chanis Águila'

${ }^{1}$ Gastroenterólogo Pediatra. Jefe del Servicio de Gastroenterología. Hospital del Niño “Dr. José Renán Esquivel”.

Correspondencia:Dr.Ricardo Chanis Águila. / Correo electrónico: rchanis0531@gmail.com

Recibido: 6 de enero de 2021

Aprobado: 5 de marzo de 2021

Publicado: 24 de junio de 2021.

DOI: $10.37980 /$ im.journal. rspp.20211707

Palabras clave: celíaco, gluten, transglutaminasa

Keywords: celiac, gluten, transglutaminase

Reproducción: Artículo de acceso libre para uso personal e individual. Sujeto a derechos de reproducción para otros usos.

Conflictos de interés: Los autores declaran no tener conflictos de interés.

Financiamiento: El autor no declara fuentes externas de financiamiento asociados a este trabajo.

\section{Resumen}

Introducción: La enfermedad celíaca es un proceso sistémico de carácter inmunológico, desencadenado por el consumo de gluten, que se da en sujetos genéticamente predispuestos. Es una condición prevalente a nivel mundial con síntomas comunes con otras patologías gastrointestinales y no gastrointestinales. En Panamá es una entidad conocida pero poco estudiada. Materiales y Método: El diseño del estudio fue observacional, descriptivo y de carácter retrospectivo en población pediátrica y adulta de la República de Panamá en unidades de gastroenterología públicas y privadas que proporcionaron sus archivos clínicos recolectados en un documento elaborado para uniformidad de las variables. Resultados: Se analizó la información de 171 expedientes. Se dividió la información en pacientes pediátricos y pacientes adultos encontrando 68 casos menores de 15 años y 103 casos mayores de 15 años. La proporción de casos masculinos y femenino fue de 1:1. De la totalidad de casos estudiados, la mayoría eran de la ciudad de Panamá en un $77 \%$ y de los extranjeros diagnosticados la población israelí fue la más frecuentemente encontrada en un $76 \%$. Los síntomas gastrointestinales más frecuentemente encontrados fueron la distensión abdominal, diarrea crónica y pérdida de peso respectivamente. La dermatitis herpetiforme, patología tiroidea y las aftas orales fueron las condiciones no gastroenterológicas más frecuentemente encontradas. La Diabetes mellitus tipo I fue la patología encontrada que coexistió con enfermedad celíaca. Conclusión: Con este estudio de carácter epidemiológico, se ha demostrado que la enfermedad celíaca es prevalente en nuestro medio sin poder mostrar un porcentaje específico.

\section{Abstract}

Introduction: Celiac disease is a systemic process of an immunological nature, triggered by the consumption of gluten, which occurs in genetically predisposed subjects. It is a prevalent condition worldwide with symptoms common with other gastrointestinal and non-gastrointestinal pathologies. In Panama it is a known but little studied entity. Method and materials:The study design was observational, descriptive, and retrospective in pediatric and adult population of the Republic of Panama in public and private gastroenterology units that provided their clinical files collected in a document prepared for uniformity of the variables. Results: Information from 171 files that met the inclusion criteria was analyzed. The information was divided into pediatric patients and adult patients, finding 68 cases under 15 years of age and 103 cases over 15 years of age. The ratio of male and female cases was 1: 1. Of the totality of cases studied, the majority were from Panama City in $77 \%$ and of the foreigners diagnosed, the Israeli population was the most frequently found in $76 \%$. The most frequently encountered gastrointestinal symptoms were abdominal distension, chronic diarrhea, and weight loss, respectively. Dermatitis herpetiformis, thyroid disease, and oral thrush were the non-gastroenterological conditions most frequently encountered. Type I Diabetes mellitus was the pathology found that coexisted with celiac disease. Conclusion: With this epidemiological study, it has been shown that celiac disease is prevalent in our environment without being able to show a specific percentage. 


\begin{tabular}{|c|c|c|c|}
\hline Edad de Diagnóstico & Fecha (año) de diagnóstico & $\begin{array}{l}\text { Sexo } \\
\text { Masculino () }\end{array}$ & Femenino () \\
\hline $\begin{array}{l}\text { Manifestaciones } \\
\text { Gastrointestinales }\end{array}$ & $\begin{array}{l}\text { Manifestaciones extra- } \\
\text { gastrointestinales }\end{array}$ & $\begin{array}{l}\text { Resultado de Biopsia } \\
1 .\end{array}$ & 2. \\
\hline & & 3. & \\
\hline $\begin{array}{l}\text { - Diarrea crónica () } \\
\text { - Pérdida de peso () } \\
\text { - Anorexia () } \\
\text { - Falla para crecer () } \\
\text { - Dolor abdominal () } \\
\text { - Vómito () } \\
\text { - Irritabilidad () } \\
\text { - Constipación () } \\
\text { - Otros: }\end{array}$ & $\begin{array}{l}\text { - Dermatitis herpetiforme () } \\
\text { - Diabetes tipo I () } \\
\text { - Hepatitis autoinmune () } \\
\text { - Alt. Del esmalte dental () } \\
\text { - Carcinoma () }\end{array}$ & $\begin{array}{l}\text { - Tiroiditis autoinmune }() \\
\text { - Artritis reumatoide }() \\
\text { - Alteración SNC } \\
\text { calcificaciones }() \\
\text { - Tumores malignos } \\
\text { linfáticos }() \\
\text { - Estomatitis recurrente }() \\
\text { - Talla baja }() \\
\text { - Otros síndromes } \\
\text { asociados }()\end{array}$ & \\
\hline $\begin{array}{l}\text { Serología: } \\
\text { 1. Antigliadina (ELISA } \\
\text { - IgG e IgA) }\end{array}$ & $\begin{array}{l}\text { 2. Antiendomisio (IFI) IgG } \\
\operatorname{IgA}\end{array}$ & $\begin{array}{l}\text { 3. Transglutaminasa IgG } \\
\text { IgA }\end{array}$ & 4. Otros (HLA) \\
\hline $\begin{array}{l}\text { Otros miembros de la } \\
\text { familia con Enf. Celiaca? }\end{array}$ & $\begin{array}{l}\text { 1er Grado () } \\
\text { 2do Grado () }\end{array}$ & & \\
\hline Residencia & Interior? & Fuera del país? & \\
\hline
\end{tabular}

\section{Introducción}

La enfermedad celíaca (EC) en una enfermedad intestinal (enteropatía mediada inmunológicamente) que afecta el intestino delgado en niños y adultos predispuestos genéticamente, desencadenada por la ingestión de alimentos que contienen gluten.

El gluten es la fracción proteica soluble (prolaminas) del trigo, centeno, cebada (avena), o de sus variedades híbridas, a las cuales algunas personas son intolerantes.

La prevalencia ha variado en los últimos 40 años, se estima que en Europa oscila entre 1 en 130 - 300 en Estados Unidos alrededor de 1: 111 - 250 encontrando una prevalencia mundial de 1:266, siendo una condición muy prevalente por lo que estimaríamos unos 15,040 casos en Panamá.

El reconocimiento de las variables clínicas, mejores pruebas de laboratorios, estudio en población de riesgo y grupos in- teresados en la patología, ha llevado a cambiar la prevalencia de la enfermedad.

En la actualidad no existen estudios epidemiológicos de prevalencia de EC en nuestro medio que se adecuen a los estándares metodológicos internacionales, es por esto por lo que se decidió realizar un estudio para describir las características epidemiológicas de los pacientes con diagnóstico confirmado de enfermedad celíaca en unidades de gastroenterología como de pacientes captados por la Fundación de Celíacos de Panamá.

\section{Material y método}

Objetivo: describir las características demográficas y clínicas de la enfermedad celiaca en Panamá.

Diseño: Estudio observacional, descriptivo, retrospectivo. 
Gráfica 1. Distribución de casos de Enfermedad Celíaca según edad y año de diagnóstico. Año 1997-2015.

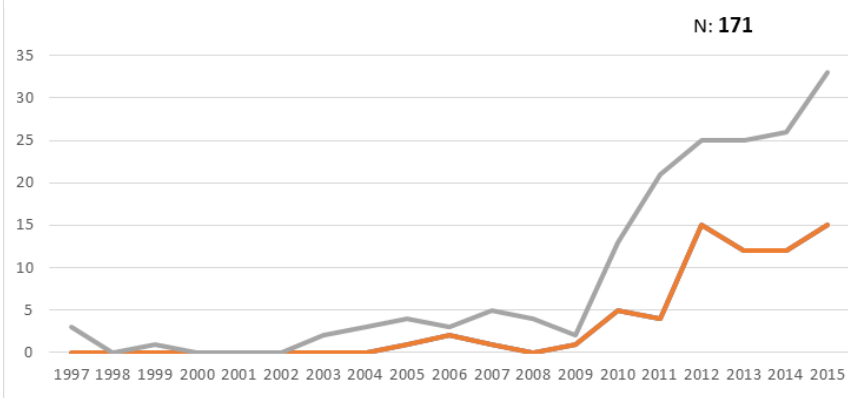

- MENORDE15AÑOS _- MAYORDE15 AÑOS

Gráfica 2. Distribución de Casos de Enfermedad Celíaca según edad y sexo. Año 1997-2015

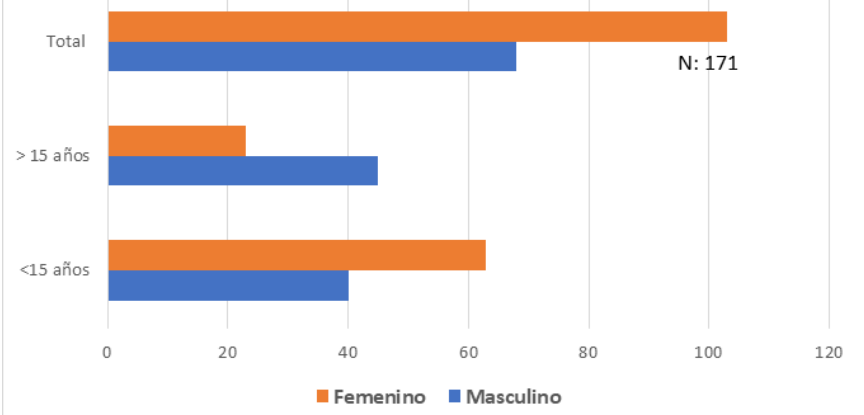

Población de estudio: masculina y femenina de todas las edades con diagnóstico confirmado de enfermedad celíaca en unidades de gastroenterología públicas y privadas, como también de la Fundación de Celíacos de la República de Panamá, años 1997 a diciembre 2015.

Criterios de inclusión: pacientes con diagnóstico de enfermedad celíaca documentado por prueba serológica lgA, lgG transglutaminasa más biopsia duodenal compatible con EC y que respondieron a la dieta sin gluten.

Criterios de exclusión: pacientes con diagnóstico de enfermedad celíaca que no contaban con prueba serológica IgA, IgG transglutaminasa o biopsia duodenal compatible con EC o que no respondieron a la dieta sin gluten.

Reclutamiento: Los datos fueron recolectados de los archivos clínicos de la consulta particular de gastroenterólogos pertenecientes a la Asociación Panameña de Gastroenterología, que aceptaron proporcionar los datos o revisar los expedientes guardando la confidencialidad de estos, así como de los archivos clínicos suministrados por la Fundación de Celíacos igualmente guardando la confidencialidad de los datos que cumplieran los criterios de inclusión.
Gráfica 3. Según nacionalidad. Año 1997-2015.

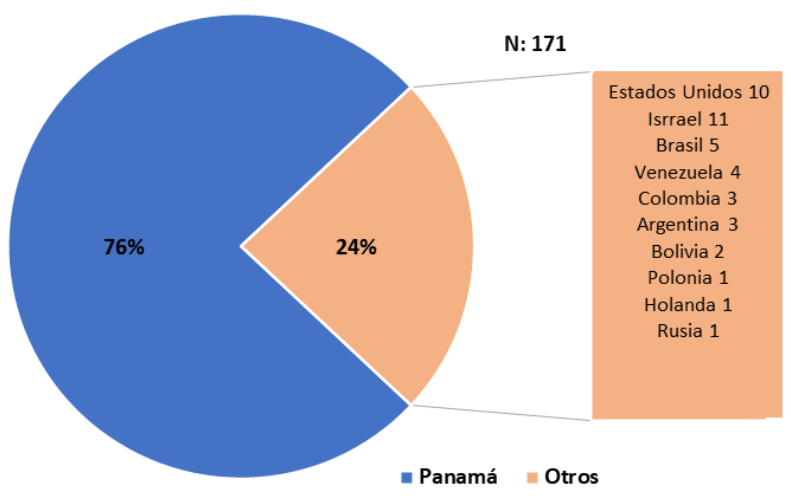

Gráfica 4. Distribución de casos de Enfermedad Celiaca según lugasr de residencia en Panamá. Año 1997-2015

N.171

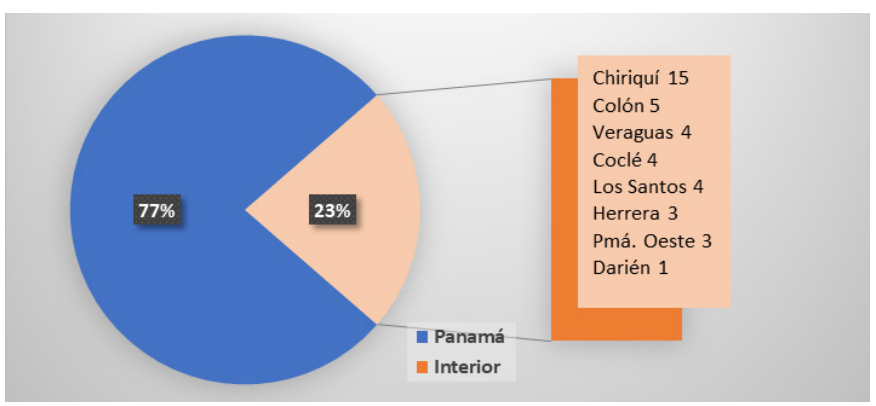

La recolección fue proporcionada de la consulta clínica del gastroenterólogo o en la fundación de Celíacos, llenando el documento elaborado para la ejecución de la información clínica y uniformar las variables a estudiar por el médico encargado del caso por un lado o por el investigador que revisó el expediente clínico. Se dividieron los expedientes en población menor de 15 años que es el criterio etario para decidir la consulta pediátrica en los hospitales pediátricos y mayores de 15 años que es la edad que atiende población adulta en los hospitales estatales de Panamá.

Las variables fueron sometidas a estadísticas descriptivas. Las cualitativas se expresaron en porcentajes y las cuantitativas en medidas de desviación estándar. Los datos fueron almacenados y las tablas y graficas elaboradas del programa Excel Microsoft Office 365.

\section{Resultados}

Se revisaron 230 expedientes clínicos de los cuales 59 no cumplían los criterios de inclusión y 171 fueron analizados para el estudio.

Las edades fueron divididas en menores y mayores de 15 años, de estos 68 casos correspondieron a menores de 15 años y 103 casos a mayores de 15 años. 
Tabla 1. Manifestaciones gastrointestinales de los pacientes con Enfermedad Celíaca según edad. Año 1997-2015

\begin{tabular}{lccc}
\hline $\begin{array}{l}\text { Manifestaciones } \\
\text { Gastrointestinales }\end{array}$ & $\begin{array}{c}<\mathbf{1 5} \text { años } \\
\text { (n: } \mathbf{6 8})\end{array}$ & $\begin{array}{c}>\text { 15 años } \\
\text { (n: 103) }\end{array}$ & $\begin{array}{c}\mathbf{N = 1 7 1} \\
\text { (\%) }\end{array}$ \\
\hline Distensión abdominal & 41 & 70 & $64 \%$ \\
Diarrea crónica & 35 & 53 & $51 \%$ \\
Pérdida de peso & 27 & 41 & $40 \%$ \\
Dolor abdominal & 21 & 32 & $31 \%$ \\
Pérdida de apetito & 18 & 28 & $27 \%$ \\
$\begin{array}{l}\text { Elevación de enzimas } \\
\text { hepáticas }\end{array}$ & 10 & 15 & $15 \%$ \\
Falla para crecer & 20 & 0 & $12 \%$ \\
$\begin{array}{l}\text { Constipación } \\
\begin{array}{l}\text { Otros síntomas } \\
\text { dispépticos }\end{array}\end{array}$ & 7 & 10 & $10 \%$ \\
\hline
\end{tabular}

La distribución de casos en los menores de 15 años ( 1 año a 14 años con 11 meses) fue en promedio de 6.4 años \pm 4.1 y en los mayores de 15 años ( 17 a 85 años) fue en promedio de $49.2 \pm 16.0$. Al no haber una concentración de edad no muy bien establecida sobre todo en los mayores de 15 años, se nota una desviación estándar muy amplia.

Antes del 2005, no había institución que hiciera las pruebas para diagnóstico de enfermedad celíaca y las mismas eran enviadas a instituciones fuera del país. No es hasta el año 2005 que el Hospital Nacional incorpora la prueba de Anticuerpo anti-transglutaminasa y comienza a observarse la aparición de los primeros casos en edades menores de 15 años. Para el año 2010 el Complejo Metropolitano de la Caja del Seguro Social, inició la realización de las pruebas y se notó un incremento en la curva de casos de EC para ambas edades. En el año 2013, se implementó la elaboración de las pruebas en el Hospital del Niño ,mostrando igualmente tendencia a la elevación en la curva de casos de EC hasta el 2015.

La proporción general de casos (171) en relación con el sexo fue M: F de 1:1. En los mayores de 15 años se observó mayor proporción de casos del sexo masculino respecto a los femeninos en una proporción de 1.9:1, distinto a los menores de 15 años donde se encontró mayor proporción de casos del sexo femenino respecto a los masculinos en 1.5:1.

De la totalidad de casos, la nacionalidad panameña fue la más frecuente con un $76 \%$ (130 casos) y un $24 \%$ (41 casos) de otras nacionalidades, donde la israelí y estadounidense predominaron y todo esto relacionado al lugar donde se realizó la investigación o sea la ciudad de Panamá y a la alta población israelí y estadounidense residente en nuestro país.

El $77 \%$ de la totalidad (132 casos) residían en la provincia de Panamá y el 23\% (39 casos) en el interior del país.

De la provincia de Chiriquí vinieron la mayor cantidad de ca-
Tabla 2. Manifestaciones no gastrointestinales de los pacientes con Enfermedad Celíaca según edad. Año 1997-2015.

\begin{tabular}{lccc}
$\begin{array}{l}\text { Manifestaciones } \\
\text { No gastrointestinales }\end{array}$ & $\begin{array}{c}<\mathbf{1 5} \text { años } \\
\text { (n: } \mathbf{6 8})\end{array}$ & $\begin{array}{c}>\mathbf{1 5} \text { años } \\
\text { (n: 103) }\end{array}$ & $\begin{array}{c}\mathbf{N}: \mathbf{1 7 1} \\
\text { (\%) }\end{array}$ \\
\hline $\begin{array}{l}\text { Dermatitis } \\
\text { Herpetiforme }\end{array}$ & 0 & 14 & $8 \%$ \\
Patología Tiroidea & 6 & 8 & $8 \%$ \\
Aftas orales & 5 & 7 & $7 \%$ \\
Anemia & 3 & 5 & $5 \%$ \\
Artralgia & 1 & 5 & $4 \%$ \\
Dermatitis atópica & 2 & 2 & $2 \%$ \\
Urticaria crónica & 1 & 3 & $2 \%$ \\
Problemas de atención & 1 & 1 & $1 \%$ \\
escolar & & & \\
Irritabilidad & 1 & 0 & $0.50 \%$ \\
Depresión & 0 & 1 & $0.50 \%$ \\
\hline
\end{tabular}

Tabla 3. Coexistencia de Enfermedad Celíaca con otras entidades inmunológicas. Año 1997-2015

\begin{tabular}{lccc}
\hline $\begin{array}{l}\text { Asociación } \\
\text { Con otras } \\
\text { entidades }\end{array}$ & $\begin{array}{c}<15 \text { años } \\
\text { (n: 68) }\end{array}$ & $\begin{array}{c}>15 \text { años } \\
\text { (n: 68) }\end{array}$ & $\begin{array}{c}\text { N: } \mathbf{1 7 1} \\
(\%)\end{array}$ \\
\hline $\begin{array}{l}\text { Diabetes tipo I } \\
\text { Síndrome de }\end{array}$ & 3 & 5 & 5 \\
$\begin{array}{l}\text { Down } \\
\begin{array}{l}\text { Enfermedad de } \\
\text { Crohn }\end{array}\end{array}$ & 0 & 0 & 2 \\
\hline
\end{tabular}

sos y pudo estar relacionado a las unidades de gastroenterología tanto adulta y pediátrica existentes en dicha provincia como también a la Fundación de Celíacos de Chiriquí.

Los síntomas y signos típicos de malabsorción (distensión abdominal, diarrea crónica y pérdida de peso), representó un importante número de casos en ambos grupos de edades y síntomas menos específicos como síntomas dispépticos se encontró de una manera importante en ambos grupos de edades.

Las manifestaciones no gastrointestinales, muchas de ellas condiciones de carácter inmunológico que pueden coexistir o ser parte de la enfermedad celíaca, ejemplo de esto; la dermatitis herpetiforme considerada como manifestación sine qua non de enfermedad celíaca que se diagnóstica con biopsia de piel. Las aftas orales a repetición signo común y la anemia por deficiencia de hierro relacionada a la malabsorción por inflamación intestinal.

La enfermedad celíaca, al ser un proceso sistémico de carácter inmunológico, es parte o coexiste con otras entidades inmunológicas como la diabetes tipo l, el síndrome de Down y las enfermedades inflamatorias intestinales.

\section{Discusión}

Este trabajo inició por la preocupación por pacientes con una sintomatología que pudiera sugerir cualquier otra entidad, resultaban en el camino tener la enfermedad celíaca. Si 
se hace un recuento histórico, antes del 2005 los casos eran esporádicos y si se sospechaba, no se tenían las facilidades diagnósticas y en aquellos en que se podía, se realizaba la prueba fuera del país. Es gracias al conocimiento de la entidad por un lado y al desarrollo de mejores métodos diagnóstico por otro lado, esta entidad que tiene más de 60 años de estar estudiándose con prevalencias altas en países suramericanos y del continente europeo, en nuestro medio a pesar de ser una entidad con un denominador común que es gluten no hay reportes de estudios epidemiológicos que resalten las características clínicas y demográficas. Esto probablemente resulta por lo difícil de recolectar la información porque muchos casos no cumplen criterios diagnósticos, no hay uniformidad en reportes histológicos y muchos otros casos se autoproclaman enfermos celiacos porque el quitar el gluten de su alimentación los hacen sentir bien.

Como definición la enfermedad celíaca (EC) es un proceso sistémico de carácter inmunológico, desencadenado por el consumo de gluten y de otras prolaminas relacionadas (secalinas, hordeínas y posiblemente aveninas) que se da en sujetos genéticamente predispuestos (sistema HLA). Cursa con una combinación variable de síntomas clínicos, marcadores serológicos específicos, haplotipo HLA-DQ2/DQ8 y enteropatía ${ }^{1,2}$.

La epidemiologia de la enfermedad celíaca ha variado en los últimos 30 años y esto es debido al reconocimiento de las variables clínicas de la enfermedad (iceberg celíaco), a mejores pruebas de laboratorio tanto inmunológicas como genéticas, al reconocimiento de los grupos de alto riesgo, nuevos conceptos histopatológicos y de las manifestaciones endoscópicas y por último y no menos importantes grupos interesados por la enfermedad que mantienen el dinamismo y la actualización de esta entidad.

En Europa se menciona una prevalencia de 1 en 300 y en Estados Unidos de 1: 111-250. Se estima que la prevalencia mundial es de 1:266 entonces es de esperar que en Panamá debiese haber unos 15,038 pacientes celíacos.

Como antecedentes de importancia en el año 2007 en la Revista Pediátrica de Panamá, Chanis R. y col publica el artículo titulado "Enfermedad Celíaca: Baja prevalencia o enfermedad subclínica, reporte de dos casos" siendo el primer artículo referente a enfermedad celíaca en Panamá. En este artículo se concluye que un mejor conocimiento de la variabilidad clínica de esta entidad al igual que el contar con mejores pruebas e instituciones de salud que realizaran las mismas daría inicio a nuevos reportes de casos 3 . Para ese entonces la única institución de carácter privado que realizaba la prueba de anticuerpo anti-transglutaminasa era el Hospital Nacional, posterior a esta en el 2010 el Complejo Hospitalario Dr. Arnulfo Arias Madrid incorpora la prueba y no es hasta el 2013 que se realiza la misma en el Hospital del Niño de Panamá.
En el 2007 las licenciadas Blake Elena y Camarena Elena elaboran el trabajo de investigación:"Frecuencia de anticuerpos anti-transglutaminasa tisular humana en pacientes con sospecha o clínica sugestiva de enfermedad celíaca referidos a la sección de inmunología del Hospital Nacional", trabajo de investigación para optar por el título de licenciada en Tecnología Médica bajo la tutoría de la Licenciada Fátima Vargas.

Encuentran en 50 sujetos, un $6 \%$ de positividad del anticuerpo anti-transglutaminasa, siendo este porcentaje elevado comparado con reportes internacionales de alrededor de un $1-2 \% .{ }^{4}$

El $1 \%$ de la población mundial padece la enfermedad y se estima que la mayoría no lo sabe. Es ligeramente más prevalente entre mujeres que entre hombres (2:1). y la edad de aparición es a los 9 años y en la cuarta de cada de la vida.

En nuestro estudio se encontró una aparición diagnóstica en promedio de 6 años en población pediátrica y de 49 años en población adulta.

En Latinoamérica existe diferencias según el área de población que se analice.

Los países en América que reportan estudios de prevalencia de EC son Estados Unidos, México, Brasil y Argentina.

Fasano et al. (2003) llevaron a cabo un estudio multicéntrico donde estudiaron la prevalencia en Estados Unidos analizando distintos grupos de población. Los resultados obtenidos señalan una prevalencia de 1:22 en familiares de primer grado, 1:39 en familiares de segundo grado, 1:56 en pacientes sintomáticos y 1:133 en el resto de la población sin riesgo. ${ }^{5}$ En un estudio publicado por la Dra. Mora M y col. siendo el primer estudio de tipo poblacional de prevalencia de enfermedad celíaca en edad pediátrica en la Argentina, la prevalencia hallada fue de 1,26\% (1/79 niños) diagnosticados por histopatología, que es más alta que la esperada pero concordante con otros estudios publicados. ${ }^{6}$

La prevalencia de la enfermedad celíaca en la población mexicana en un estudio de Aguilar-Olivos fue mayor a la prevalencia global mundial reportada de $2.17 \%$.

La enfermedad celíaca es subdiagnosticada debido a la heterogeneidad de su forma de presentación, la sintomatología puede ser muy variable de unas personas a otras y diferir según la edad. Es importante tener en cuenta que hay un alto porcentaje de personas, tanto niños como adultos, que presentan síntomas atípicos o están asintomáticos, lo que dificulta el diagnóstico.

El espectro de síntomas y signos es amplio en pacientes al momento del diagnóstico de EC. Los síntomas clásicos de 
malabsorción parecen ser más específicos e incluyen retraso del crecimiento, pérdida de peso y diarrea crónica tal como se evidencia en esta revisión. Para síntomas menos específicos, existe evidencia de que los pacientes con síntomas similares al SII (síndrome de intestino irritable) con predominio de diarrea, anemia por deficiencia de hierro, estreñimiento crónico y defectos del esmalte tienen un mayor riesgo de EC. Para otros síntomas gastrointestinales inespecíficos como dolor abdominal, dispepsia e distensión abdominal, no hay evidencia suficiente. ${ }^{8-12}$

El diagnóstico está basado en la sospecha clínica, la confirmación de pruebas estandarizadas y la respuesta a la eliminación del gluten en la alimentación.

En cuanto a las pruebas, la anti-gliadina que apareció a principios de 1980 como una prueba de oro en el diagnóstico de enfermedad celíaca, ha caído en desuso porque el conocimiento de la evolución, y mecanismos inmunológicos de producción de enfermedad ha dado lugar a nuevas y mejores pruebas y rutas diagnósticas para evitar diagnósticos errados por un lado o sobre diagnóstico por el otro lado.

Para la prueba inicial, la combinación de anticuerpos de clase IgA e lgG totales contra la transglutaminasa 2 (TGA-lgA) es más precisa que otras combinaciones de pruebas.

El enfoque sin biopsia para el diagnóstico de EC es seguro en niños con niveles altos de TGA-lgA valores ( $\geq 10$ veces el límite superior de la normalidad) con pruebas apropiadas y endomisio positivo, anticuerpos (EMA-IgA) en una segunda muestra de suero. Niños con TGA-lgA positivo, pero títulos más bajos ( $<10$ veces el límite superior de lo normal), deben someterse a biopsias para disminuir el riesgo de diagnóstico falso positivo. ${ }^{13-15}$

Las pruebas de HLA aún con presencia de síntomas no es criterio diagnóstico único, sin una serología positiva y diagnóstico basado en biopsia intestinal. ${ }^{16,17}$

Hay personas que tienen más riesgo de desarrollar la enfermedad, sobre todo las que tienen familiares de primer grado afectos o las que padecen otras enfermedades en las que interviene el sistema inmune como la diabetes, enfermedad inflamatoria intestinal. Los niños con enfermedades cromosómicas (como el síndrome de Down, Williams, Turner entre otros), intolerancia a lactosa primaria genéticamente predeterminada o déficit selectivo de inmunoglobulina $A$ también tienen más riesgo de padecer enfermedad celiaca, al igual que otras entidades como enfermedad hepática y tiroidea autoinmune. ${ }^{18,19}$

En este momento, el diagnóstico de la enfermedad está basado en 4 pilares fundamentales que se combinan entre sí para llegar al diagnóstico.
Presencia o ausencia de síntomas o signos compatibles con la enfermedad, elevación en sangre de títulos de anticuerpos específicos de enfermedad celiaca. Se tiene en cuenta tanto el tipo de anticuerpo estudiado, como los niveles obtenidos. Cada uno tiene sus particularidades, que hay que conocer para poder interpretarlos de forma adecuada.

Estudio de predisposición genética con la determinación en sangre de los haplotipos de HLA DQ2 y DQ8, apoya el diagnóstico, pero no lo confirma, ya que hasta el $30 \%$ de la población sana es portadora de los genes y sólo el $1 \%$ de ellos son celíacos. Actualmente no es necesaria su determinación para la confirmación del diagnóstico en los casos que cumplan ciertos criterios de anticuerpos positivos, lo cual será valorado por el especialista en gastroenterología pediátrica. ${ }^{20-26}$

La biopsia intestinal sigue siendo el estándar de oro y su realización dependerá de los criterios descritos anteriormente. La inflamación pudiera estar en parches en la mucosa intestinal por lo que para optimizar su estudio por un entero patólogo con conocimiento de la entidad se deben obtener al menos 4 biopsias de duodeno y por lo menos una de bulbo duodenal ya que, en ocasiones, esta localización pudiera ser la única con lesión.

Existe cierta dificultad en su interpretación y como consecuencia, resultados discordantes entre patólogos. Por esto es necesario, emplear métodos estandarizados para la correcta orientación y corte de la biopsia duodenal. Generalmente, se emplea la clasificación de Marsh modificada por Oberhüber para categorizar las distintas lesiones histológicas. ${ }^{27-30}$

La confirmación definitiva de la enfermedad es evolutiva, con la desaparición de los síntomas y la normalización de las alteraciones analíticas tras la retirada del gluten de la dieta. Se estima que los títulos de anti-transglutaminasa pueden desaparecer después de 6 meses de dieta estricta sin gluten y la lesión histológica recuperarse hasta después de dos años.

El conjunto de estos datos nos permite confirmar o excluir el diagnóstico en la mayoría de los casos, aunque en ocasiones es preciso un seguimiento y repetir alguno de los estudios, e incluso puede ser necesaria la realización de una prueba de provocación (analizar la respuesta a la reintroducción del gluten en la dieta durante un tiempo definido).

\section{Conclusión}

Con este estudio de carácter epidemiológico queda demostrado que la enfermedad celíaca es prevalente en nuestro medio sin poder mostrar un porcentaje específico. Hacer un diagnóstico requiere sospecharlo; confirmarlo, requiere del conocimiento de la entidad como de la ejecución adecuada y establecida de las pruebas y estudios ya avalados, consen- 
suados y siempre con el apoyo del gastroenterólogo. Diagnosticar enfermedad celíaca, involucra eliminar de por vida el gluten de la alimentación y por esto es necesario el diagnóstico fino y esto se consigue si se siguen los lineamientos diagnósticos.

La enfermedad celíaca en la "gran imitadora" comparte síntomas como muchas patologías orgánicas y funcionales por lo que hay que tenerla siempre en mente y desarrollar un adecuado abordaje diagnóstico.

\section{Referencias}

1. Downey L, Houten R, Murch S, Longson D. Recognition, assessment, and management of coeliac disease: summary of updated NICE guidance. BMJ. 2015;351:h4513. doi: 10.1136/ bmj.h4513.

2. Werkstetter KJ, Korponay-Szabo IR, Popp A, et al. ProCeDE study group. Accuracy in diagnosis of celiac disease without biopsies in clinical practice. Gastroenterology 2017; 15(4)3:924-35. doi: 10.1053/j.gastro.2017.06.002. Epub 2017 Jun 15.

3. Chanis R, Mc Calla R, Chong E, Rodríguez G, Surgeon J. Enfermedad Celiaca: ¿Baja prevalencia o enfermedad subclínica?, Reporte de dos casos y revisión de la literatura? Pediátr Panamá. 2007;36(1):96-101.

4. Blake E, Camarena E. Frecuencia de anticuerpo antitrasglutaminasa humana en pacientes con sospecha clínica de enfermedad celíaca referidos a la sección de inmunología de la Clínica Nacional. Marzo a noviembre 2007. Universidad de Panamá. Facultad de Medicina. Escuela de Tecnología médica. Grado académico para licenciatura en tecnología médica.

5. Fasano A, Berti I, Gerarduzzi T, Not T et al. Prevalence of celiac disease in at-risk and not-at-risk groups in the United States: a large multicenter study. Arch Intern Med. 2003; 163 (3): 286292. doi: 10.1001/archinte.163.3.286.

6. Mora M, Litwinb N, Toca MC, Azcona MI, Solís Neffa R, Battistona F, Solaeguia M, Ortiz G. Prevalencia de enfermedad celíaca: estudio multicéntrico en población pediátrica de cinco distritos urbanos de la Argentina Arch Argent Pediatr 2012;110(6):490496.

7. Aguilar-Olivos NE, Motola-Kuba M, Toapanta-Yanchapaxi L et al. Prevalencia de la enfermedad celíaca en una población mexicana. Med Sur. 2015;22(2):64-69.

8. Shakeri R, Zamani F, Sotoudehmanesh R et al. Gluten sensitivity enteropathy in patients with recurrent aphthous stomatitis. BMC Gastroenterol. 2009; 9:44. doi: 10.1186/1471-230X-9-44.

9. Falcon AC, Fifi AC, Fernandez-Valdes L, Jaquez J, Mariaud C, Saps M. Celiac Disease Testing Necessary in Functional Abdominal Disorders? A Study in Predominantly Latino Children. J Pediatr Gastroenterol Nutr: November 20, 2020 - Volume Publish Ahead of Print - Issue - doi: 10.1097/MPG.0000000000002993.

10. Laass MW, Schmitz R, Uhlig HH, Zimmer KP, Thamm M, Koletzko $S$. The prevalence of celiac disease in children and adolescents in Germany. Dtsch Arztebl Int. 2015;112(33- 34):553-60. doi: 10.3238/arztebl.2015.0553.

11. Kalayci AG, Kanber Y, Birinci A, Yildiz L, Albayrak D. The preva- lence of coeliac disease as detected by screening in children with iron deficiency anemia. Acta Paediatr. 2005;94(6):678- 81. doi: 10.1111/j.1651-2227.2005.tb01964.x.

12. Khatib M, Baker RD, Ly EK, Kozielski R, Baker SS. Presenting Pattern of Pediatric Celiac Disease. J Pediatr Gastroenterol Nutr. 2016;62(1):60-3. doi: 10.1097/MPG.0000000000000887.

13. Bishop J, Reed P, Austin P et al. Prospective Evaluation of the ESPGHAN Guidelines for Diagnosis of Celiac Disease in New Zealand Children. J Pediatr Gastroenterol Nutr. 2018; 67(6):749754.doi: 10.1097/MPG.0000000000002065.

14. Previtali G,Licini, L, D'Antiga L, Marseglia, A, Ravasio L, Nembrini F, Greco S; Sonzogni A; Azzarà G; Ravelli P; Alessio M. Celiac Disease Diagnosis Without Biopsy: Is a 10X ULN Antitransglutaminase Result Suitable for a Chemiluminescence Method? J Pediatr Gastroenterol Nutr. 2018;66(4):645-650. doi: 10.1097/ MPG.0000000000001773

15. Webb C, Norström F, Myléus A et al. Celiac disease can be predicted by high levels of anti-tissue transglutaminase antibodies in population-based screening. J Pediatr Gastroenterol Nutr 2015; 60(6):787-91. doi: 10.1097/MPG.0000000000000688.

16. Lopes LHC, Muniz JG, Oliveira RP, Sdepanian VL. Celiac Disease in Brazilian First-degree Relatives: The Odds Are Five Times Greater for HLA DQ2 Homozygous. J Pediatr Gastroenterol Nutr. 2019;68(5) ):e77-e80.doi: 10.1097/MPG.0000000000002251.

17. Koning F. Pathophysiology of Celiac Disease. J Pediatr Gastroenterol Nutr.2014;59:Suppl 1:S1-4.doi: 10.1097/01. mpg.0000450391.46027.48

18. Sattar N, Lazare F, Kacer M et al. Celiac disease in children, adolescents, and young adults with autoimmune thyroid disease. J Pediatr. 2011;158(2):272-5.e1. doi: 10.1016/j.jpeds.2010.08.050. Epub 2010 Oct 18.

19. Khan MR, Nellikkal SS, Barazi A, Larson JJ, Murray JA, Absah I. The Risk of Autoimmune Disorders in Treated Celiac Disease Patients in Olmsted County, Minnesota. J Pediatr Gastroenterol Nutr. 2019; 69 (4);438-442. doi: 10.1097/ MPG.0000000000002418.

20. Steinhauser S, Schumacher M, Rücker G. Modelling multiple thresholds in meta-analysis of diagnostic test accuracy studies. BMC Med Res Methodol 2016; 16(1):97. doi: 10.1186/s12874016-0196-1.

21. Husby S, Koletzko S, Korponay-Szabó I et al.European Society Paediatric Gastroenterology, Hepatology and Nutrition. Guidelines for Diagnosing Coeliac Disease 2020;70(1):141-156. doi: 10.1097/MPG.0000000000002497.

22. Vecino R, Miranda C, Rodrigo G, Romero RG, Cilleruelo ML, Román E. Influence of the 2012 European Guidelines in Diagnosis and Follow-up of Coeliac Children With Selective IgA Deficiency. J Pediatr Gastroenterol Nutr.2020;71(1):59-63. doi: 10.1097/ MPG. 0000000000002634.

23. Cilleruelo $M L$, Fernández-Fernández $S$, Jiménez-Jiménez J, Rayo Al, Hernando de Larramendi C. Prevalence and natural history of celiac disease in a cohort of at-risk children. J Pediatr Gastroenterol Nutr 2016; 62(5):739-45. doi: 10.1097/ MPG.0000000000001007.

24. Paul SP, Sandhu BK, Spray CH, Basude D, Ramani P. Evidence 
supporting serology-based pathway for diagnosing celiac disease in asymptomatic children from high-risk groups. J Pediatr Gastroenterol Nutr. 2018; 66(4):641-4. doi: 10.1097/ MPG.0000000000001757

25. Jansen $M$, van Zelm M, Groeneweg $M$ et al. The identification of celiac disease in asymptomatic children: The Generation R Study. J Gastroenterol 2018; 53(3):377-86. doi: 10.1007/ s00535-017-1354-x. Epub 2017 Jun 6

26. Villalta $D$, Tonutti $E$, Prause $C$ et al.IgG Antibodies against DeamidatedGliadin Peptides for diagnosis of Celiac Disease in Patients with IgA Deficiency. Clin Chem. 2010;56 (3):464-468. doi: 10.1373/clinchem.2009.128132. Epub 2009 Dec 18.

27. Montén C, Bjelkenkrantz K, Gudjonsdottir AH et al. Validity of histology for the diagnosis of paediatric coeliac disease: a Swedish multicentre study. Scand J Gastroenterol 2016; 51(4):42733. doi: 10.3109/00365521.2015.1101486. Epub 2015 Dec 4.
28. Villanacci V, Lorenzi L, Donato F et al. Histopathological evaluation of duodenal biopsy in the PreventCD project. An observational interobserver agreement study. APMIS 2018; 126 (3):208-14. doi: 10.1111/apm.12812. Epub 2018 Jan 26.

29. Posthumus L, Al-Toma A. Duodenal histopathology and laboratory deficiencies related to bone metabolism in coeliac disease. Eur J Gastroenterol Hepatol. 2017;29(8):897-903. doi: 10.1097/MEG.0000000000000880.

30. Adelman DC, Murray J, Wu TT, Mäki M, Green PH, Kelly Cp. Measuring change in small intestinal histology in patients with celiac disease. Am J Gastroenterol.2018; 113 (3):339-47. doi: 10.1038/ajg.2017.480. Epub 2018 Feb 20. 